\title{
Posterior Inferior Cerebellar Artery Aneurysm, Mimicking Lateral Medullary Infarction: A Case Report
}

\author{
Chang Hyeong Kim${ }^{1}$, Kyung Min $\mathrm{Yi}^{2}$ \\ ${ }^{1}$ Department of Neurology, Munsung Hospital, Daegu, Korea \\ ${ }^{2}$ Department of Nursing, Suseong University, Daegu, Korea
}

Corresponding Author:

Chang Hyeong Kim, MD

https://orcid.org/0000-0002-4364-3992

Department of Neurology, Munsung

Hospital, 168, Seongdang-ro, Namgu,

Daegu 42459, Korea

Tel: +82-53-659-7120

Fax: +82-53-653-2813

E-mail: ego0001@naver.com

Received: May 28, 2018

Accepted: June 19, 2018

\begin{abstract}
Posterior inferior cerebellar artery (PICA) aneurysm is relatively rare. We report on a 74-year-old woman presenting with dizziness and right-side tilted gait caused by a rightside distal PICA aneurysm, which mimicked a right lateral medullary infarction. A neurological examination showed right-side limb ataxia and left-beating torsional nystagmus, similar to a right lateral medullary infarction. Magnetic resonance imaging revealed a distal PICA aneurysm in the right-side middle cerebellar peduncle region. (Ann Geriatr Med Res 2018;22:102-104)
\end{abstract}

Key Words: Aneurysm, Posterior inferior cerebellar artery, Lateral medullary infarction

\section{INTRODUCTION}

Although the posterior inferior cerebellar artery (PICA) is the last and largest branch of the vertebral artery, its thin lumen makes it difficult to be observed on computed tomography angiography or magnetic resonance angiography (MRA). ${ }^{1)}$ Aneurysms of the PICA are rare, accounting for about $0.5 \%-1 \%$ of intracranial aneurysm cases. ${ }^{2)}$ PICA aneurysm can be easily missed by clinicians, leading to misdiagnosis and treatment delays. Lateral medullary infarction is a neurological disorder causing various symptoms due to ischemia in the lateral part of the medulla oblongata in the brainstem.

This study reports on the case of a patient who was initially presumed to have a lateral medullary infarction and presented with severe dizziness, ipsilateral ataxia, and gait disturbance, but was finally diagnosed with PICA aneurysm, via magnetic resonance imaging and MRA.

\section{CASE REPORT}

A 74-year-old woman visited us owing to the presence of severe rotational vertigo, right side tilting gait, and right limb ataxia for 3 days. She did not complain of dysarthria, dysphagia, and facial sensory change. She has been on hypertension medications for 3 years. She did not drink or consume alcohol. At the clinic, her vital signs were all normal. On neurologic examination, we noted impaired right side cerebellar function via the finger to nose and heel to shin test, ataxia of the right limbs, and spontaneous left side beating horizontal-torsional nystagmus.

The blood test results were normal, except for hyperlipidemia. Considering her condition and clinical findings, we presumed she had right lateral medullary infarction. Then, we performed a brain magnetic resonance imaging and MRA. Cytotoxic edematous changes were not noted on the diffusion-weighted images (Fig. 1A). T2-weighted and T2-fluid attenuated inversion recovery imaging revealed a lesion-like halo, surrounded by edema at the right cerebellar peduncle area (Fig. 1B, C). There was no lesion with contrast enhancement and the possibility of a tumor was excluded (Fig. 1D). MRA showed right distal PICA aneurysm (about $4 \mathrm{~mm}$ ) in the axial image and 3-dimensional reconstruction image (Fig. 1E, F). The patient was then referred to the neurosurgery department.

\section{DISCUSSION}

Autopsy studies have revealed that unruptured intracranial aneurysms are prevalent in $0.2 \%-9 \%$ of the population. ${ }^{3)}$ Regarding the diagnosis of intracranial aneurysms using 1.5-T MRA, a previous study reported that intracranial aneurysms were detected in $4.5 \%$ of outpatient examinees. ${ }^{4)}$ Cases of PICA aneurysm are relatively rare, accounting for $0.5 \%-1 \%$ of intracranial aneurysm cases. ${ }^{1)}$ However, it can cause brainstem lesions; therefore, it is very dangerous and can be fatal if they rupture. The most common complication of intracranial aneurysms is a rupture caus- 

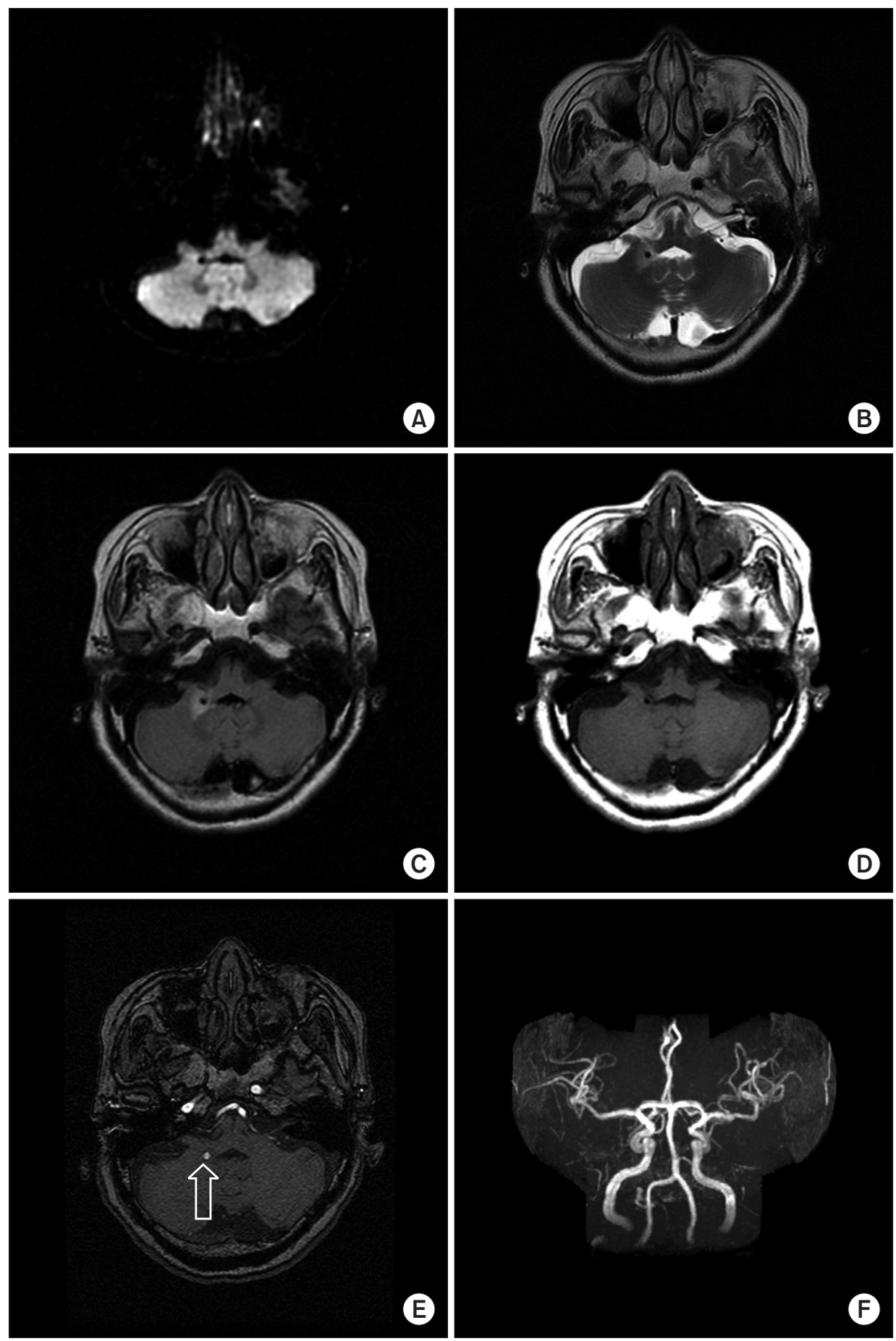

Fig. 1. Brain magnetic resonance (MR) diffusion-weighted (A), T2weighted (B), T2-fluid attenuated inversion recovery (C), and $\mathrm{T} 1$ enhancement (D) images of the axial view show a lesion-like halo, surrounded by edema at the right cerebellar peduncle area. (E, F) MR angiography revealed right distal posterior inferior cerebellar artery aneurysm (about $4 \mathrm{~mm}$ ) in the axial image (arrow) and 3-dimensional reconstruction image.

ing subarachnoid hemorrhage. An anomalous origin of the right subclavian artery and right vertebral artery is one of the etiologies of the right PICA aneurysm. One etiology of right PICA aneurysm is anomalous origins of the right subclavian artery and right vertebral artery. ${ }^{5}$ Thin axial computed tomography (CT) images are most important and reliable for the detection of distal PICA aneurysm cases. ${ }^{1)}$ Unfortunately, we did not perform carotid angiogram and CT angiogram.

In our case, the PICA aneurysm compressed the inferior cerebellar peduncle and inferior vestibular nucleus in the medulla. Therefore, it could cause ipsilateral limb ataxia and contralateral nystagmus. We believe that the relative location of the aneurysm makes that highly probable. Treatment of PICA aneurysms depends on its anatomical variations. ${ }^{6)}$

Conflicts of Interest Disclosures: The researchers claim no conflicts of interest. 


\section{REFERENCES}

1. Zhang Y, Sun S, Xu Q, Feng W, Chen H. Posterior inferior cerebellar artery aneurysm: have you ever been misdiagnosed? Acad Radiol 2018 Mar 1. pii: S1076-6332(18)30054-0. https://doi.org/ 10.1016/j.acra.2018.01.025.

2. Peluso JP, Van Rooij WJ, Sluzewski M, Beute GN, Majoie CB. Posterior inferior cerebellar artery aneurysms: incidence, clinical presentation, and outcome of endovascular treatment. Am J Neuroradiol 2008;29:86-90.

3. Housepian EM, Pool JL. A systematic analysis of intracranial an- eurysms from the autopsy file of the Presbyterian Hospital, 1914 to 1956. J Neuropathol Exp Neurol 1958;17:409-23.

4. Jeon TY, Jeon P, Kim KH. Prevalence of unruptured intracranial aneurysm on MR angiography. Korean J Radiol 2011;12:547-53.

5. Adi MY, Adams W. Right PICA aneurysm with anomalous origins of the right subclavian artery and right vertebral artery: findings on angiography. Br J of Neurosurg 2018 Feb 28 [Epub]. https:// doi.org/10.1080/02688697.2018.1444143.

6. Singh RK, Behari S, Kumar V, Jaiswal AK, Jain VK. Posterior inferior cerebellar artery aneurysms: Anatomical variations and surgical strategies. Asian J Neurosurg 2012;7:2-11. 